\title{
Capacidad Inspiratoria y Polimorfismo de la Enzima Convertidora de Angiotensina en Jóvenes Chilenos
}

\author{
Inspiratory Capacity and Polymorphism of Angiotensin Converter Enzyme in Young Chileans
}

\author{
Rodrigo Muñoz Cofréé ${ }^{1,2}$; Mariela Muñoz ${ }^{1,3}$; Daniel Conei ${ }^{1,4}$; Mariano del Sol ${ }^{1}$ \& Alejandro Pacheco Valles ${ }^{5,6}$
}

\begin{abstract}
MUÑoz, C. R.; MUÑoz, M.; CONEI, V. D.; DEL SOL, M. \& PACHECO, V. A. Capacidad inspiratoria y polimorfismo de la enzima convertidora de angiotensina en jóvenes chilenos. Int. J. Morphol., 36(3):1149-1153, 2018.

RESUMEN: El objetivo de la presente investigación fue determinar la capacidad inspiratoria (CI) en un grupo de universitarios chilenos sedentarios agrupados por el genotipo (polimorfismo rs4646994) del gen de la enzima convertidora de angiotensina (ECA). Se seleccionaron a través de muestreo no probabilístico 83 individuos (de 18 a 35 años), 37 mujeres y 46 hombres. Se midió la CI a través de pletismografía corporal, se extrajo ADN a partir de sangre con EDTA y se realizó la reacción en cadena polimerasa (PCR) para el polimorfismo Ins/Del de la ECA. Se determinó normalidad de los datos, utilizándose t de Student o ANOVA para las variables paramétricas, y en las no paramétricas U de Mann-Whitney o Kruskal-Wallis, considerándose significativo un valor de $\mathrm{p}<0,05$. La distribución de los genotipos se encontró en equilibrio de Hardy Weinberg $\left(\mathrm{X}^{2}=1,872, \mathrm{p}=0,171\right.$ para los hombres y $\mathrm{X}^{2}=3,424$, $\mathrm{p}=0,064$ para las mujeres), la CI, en ambos sexos, no mostró diferencias significativas al ser comparada por genotipo. Los hombres portadores del genotipo Ins/Ins presentaron mayores niveles de CI en relación al grupo del gentipo Del/Del-Ins/Del. Las mujeres con la dominancia alélica Ins presentaron una mayor CI que las pertenecientes al grupo Del/Del. Existió una mayor CI en participantes de sexo femenino con dominancia alélica Ins y masculino homocigotos Ins, del gen de la ECA.
\end{abstract}

PALABRAS CLAVE: Capacidad inspiratoria; Enzima convertidora de angiotensina; Sedentarismo.

\section{INTRODUCCIÓN}

La capacidad inspiratoria (CI), es una de las variables de función pulmonar que en la actualidad está adquiriendo una gran importancia en el diagnóstico de la funcionalidad en enfermedades respiratorias, debido a su directa relación con la capacidad de realizar ejercicio, aquí su disminución se correlaciona directamente con la gravedad de la enfermedad (Richter et al., 2017). La disnea generada se asocia al descenso de la CI, la cual provoca una limitación al esfuerzo físico y alteraciones en los patrones ventilatorios. Además de esto, la CI es sensible a los cambios de la presión muscular inspiratoria (PIM), al respecto se ha descrito una relación directa entre ambas variables (Pessoa et al., 2017). Si bien, las diferencias en función pulmonar, según sexo, edad y etnia están ampliamente estudiadas (Menezes et al., 2015), la influencia genética sobre la CI aún está en desarrollo.

En este contexto, el polimorfismo Inserción (Ins)/
Deleción (Del) de la enzima convertidora de angiotensina (ECA) ha demostrado influir sobre la fuerza muscular inspiratoria y espiratoria (Muñoz et al., 2017). Al respecto, se sabe que la actividad enzimática y la producción de energía varían en un mismo grupo muscular entre distintos sujetos, lo que tendría relación con la predominancia de polimorfismos específicos (Caló \& Vona, 2008). El polimorfismo Ins/Del del gen de la ECA se asocia a variaciones en la regulación del volumen sanguíneo, presión arterial y función vascular, factores que impactan en el rendimiento muscular entre distintos individuos (Nguyen Dinh Cat \& Touyz, 2001).

Considerando la directa relación existente entre la PIM y la CI, hipotetizamos que existiría un aumento en la CI en los sujetos con Ins del gen de la ECA. Así, el objetivo del presente estudio fue determinar la CI en un grupo de universitarios chilenos categorizados por el genotipo (polimorfismo rs4646994) del gen de la ECA.

\footnotetext{
${ }^{1}$ Programa de Doctorado en Ciencias Morfológicas, Universidad de La Frontera, Temuco, Chile.

${ }^{2}$ Laboratorio de Función Disfunción Ventilatoria Universidad Católica del Maule, Talca, Chile.

${ }^{3}$ Laboratorio de Morfología Molecular, Centro de Estudios Morfológicos y Quirúrgicos, Universidad de La Frontera, Temuco, Chile.

${ }^{4}$ Departamento de Ciencias Morfológicas, Facultad de Ciencia, Universidad San Sebastián, Puerto Montt, Chile.

${ }^{5}$ Departamento de Kinesiología, Facultad de Ciencias de la Salud, Universidad Católica del Maule, Talca, Chile.

${ }^{6}$ Programa de Magíster en Kinesiología, Universidad Católica del Maule, Talca, Chile.
} 


\section{MATERIAL Y MÉTODO}

La muestra correspondió a 83 estudiantes de la Universidad Católica del Maule de 18 a 35 años de edad (Muñoz et al.) seleccionados a través de un muestreo no probabilístico, aceptaron voluntariamente formar parte de este estudio, el cual fue aprobado por el Comité de Ética Científica de la Universidad Católica del Maule (23/2016). Se excluyeron aquellos sujetos con antecedentes de enfermedad respiratoria crónica, hábito tabáquico, deformidad torácica, volumen espirado al primer segundo $\left(\mathrm{VEF}_{1}\right)$ menor al $80 \%$ del predicho, sobrepeso (IMC $\geq 24,9 \mathrm{~kg} / \mathrm{m}^{2}$ ), relación consanguínea con otro participante y deportistas.

Evaluación de la función pulmonar. Las pruebas se realizaron en un pletismógrafo corporal (Mediagraphics Modelo PlatinumElite DL $\left.{ }^{\circledR}\right)$. Se midió capacidad vital forzada (CVF) y CI según la normativa de la American Thoracic Society (ATS) (ATS, 2005).

\section{Análisis Genético}

Extracción de ADN: se extrajo a partir de $5 \mathrm{~mL}$ de sangre anticoagulada con ácido etilendiaminotetraacético (EDTA), mediante el micrométodo de yoduro de sodio de Salazar $e t$ al. (1998). El ADN extraído se guardó a $-20^{\circ} \mathrm{C}$. La integridad del ADN extraído, se evaluó mediante electroforesis en gel de agarosa al $2 \%$, teñido con Gel Red® (Biotium, Hayward, CA, E.E.U.U.), se cuantificó a través de espectrofotometría a $260 \mathrm{~nm}$.

Diseño de partidores: Se seleccionó como secuencia de interés la región comprendida entre la posición 11.332 y 11.643 del gen de la ECA, lo que flanqueaba por ambos lados la región de Ins/Del del intrón 16. Para el diseño de los partidores se utilizó el software online Primer3 v0.4.0 (disponible en http://frodo.wi.mit.edu/). La secuencia de los partidores diseñados fueron P1: 5'CTGGAGAGCCACTCCCATCCTTTCT-3' y P2: 5'GACGTGGCCATCACATTCGTCAGAT-3'. Para el genotipo Ins/Ins se obtiene una única banda de $479 \mathrm{pb}$, para el genotipo Ins/Del dos bandas de 192 pb y 479 pb y para el Del/Del una única banda de $192 \mathrm{pb}$.

Amplificación de ADN: Se realizó un master mix con tampón de PCR 1x, $2 \mathrm{mM}$ de cloruro de magnesio $\left(\mathrm{MgCl}_{2}\right), 0,2$ $\mathrm{mM}$ de cada dNTP, $0,5 \mathrm{U} / \mathrm{mL}$ de Taq DNA polimerasa (Fermentas, Lituania), $200 \mathrm{nM}$ de cada partidor, $100 \mathrm{ng}$ de ADN y agua desionizada estéril para un volumen final de $20 \mathrm{~mL}$. Se corrió el siguiente programa de PCR, desnaturalización inicial a $95{ }^{\circ} \mathrm{C}$ por $5 \mathrm{~min}$, luego 32 ciclos de $95{ }^{\circ} \mathrm{C}$ por 30 segundos, $60{ }^{\circ} \mathrm{C}$ por 30 segundos y $72{ }^{\circ} \mathrm{C}$ por $30 \mathrm{~s}$, y una extensión final a $72{ }^{\circ} \mathrm{C}$ por $10 \mathrm{~min}$ en equipo termociclador T100 (Bio Rad, USA). Se evaluaron los productos de la PCR mediante electroforesis en gel de agarosa al $2 \%$ teñido con Gel Red® (Biotium, Hayward, CA, E.E.U.U.) (Rosales et al., 2009).

Análisis Estadístico. Para el análisis estadístico, los datos se tabularon y analizaron en los programas Microsoft Office Excel 2013 y GraphPad Prism 5®, respectivamente. Los resultados se presentan como promedios y desviación estándar. Se determinó la normalidad en la distribución de los datos, posteriormente se usó la prueba t de Student o ANOVA según corresponda, las mediciones que no cumplieron una distribución paramétrica se analizaron con la prueba U de Mann-Whitney o Kruskal-Wallis. Se consideró un nivel de significancia de $\mathrm{p}<0,05$.

Tabla I. Características generales de jóvenes sedentarios de la Universidad Católica del Maule, Chile.

\begin{tabular}{lccc}
\hline Variable & $\begin{array}{c}\text { Sexo Femenino } \\
(\mathrm{n}=37)\end{array}$ & $\begin{array}{c}\text { Sexo Masculino } \\
(\mathrm{n}=46)\end{array}$ & Valor $p$ \\
& Promedio $\pm \mathrm{DE}$ & Promedio $\pm \mathrm{DE}$ & \\
& $21,81 \pm 1,39$ & $23,57 \pm 3,03$ & $0,001^{\S}$ \\
Edad (años) & $59,63 \pm 8,79$ & $74,76 \pm 10,35$ & $0,0001^{\S}$ \\
Peso (kg) & $1,61 \pm 0,06$ & $1,73 \pm 0,07$ & $0,0001^{\S}$ \\
Talla (m) & $23,01 \pm 2,84$ & $24,80 \pm 2,88$ & $0,004^{£}$ \\
IMC (kg/m $\left.{ }^{2}\right)$ & $3,91 \pm 0,51$ & $5,46 \pm 0,58$ & $0,0001^{£}$ \\
CVF (L) & 109,00 & 110,00 & - \\
CVF (\% predicho) & $3,37 \pm 0,38$ & $4,56 \pm 0,48$ & $0,0001^{£}$ \\
VEF $(\mathrm{L})$ & 97,00 & 107 & - \\
VEF $(\%$ predicho) & $118,62 \pm 35,40$ & $163,85 \pm 35,82$ & $0,0001^{£}$ \\
CI $(\mathrm{L})$ & 92,00 & 126,00 & - \\
CI $(\%$ predicho $)$ & & \\
\hline
\end{tabular}

DE: desviación estándar; IMC: índice de masa corporal; kg: kilogramo; s: segundos; CVF: capacidad vital forzada; $\mathrm{VEF}_{1}$ : volumen espiratorio forzado al primer segundo; CI: capacidad inspiratoria; (§): Mann-Whitney; (£): t-student. 


\section{RESULTADOS}

La distribución de los genotipos se encontró en equilibrio de Hardy Weinberg; $\mathrm{X}^{2}=1,872$, $\mathrm{p}=0,171$ para los hombres y $\mathrm{X}^{2}=3,424, \mathrm{p}=0,064$ para las mujeres. En la Tabla I se observa el comportamiento antropométrico y espirométrico de todos los participantes, los cuales se encuentran dentro de los rangos de normalidad. En ambos sexos la CI no mos- tró diferencias significativas al compararla por genotipo (Tabla II). Los hombres portadores del genotipo Ins/Ins presentaron una mayor CI en relación al grupo Ins/Del-Del/ Del (Tabla III). Del mismo modo, las mujeres con la dominancia alélica Ins presentaron una mayor CI que las pertenecientes al grupo Del/Del (Tabla III).

Tabla II. Capacidad Inspiratoria divida por genotipo en jóvenes sedentarios de ambos sexos de la Universidad Católica del Maule, Chile.

\begin{tabular}{lccccccc}
\hline \multirow{2}{*}{ Variable } & \multicolumn{3}{c}{ Sexo Femenino } & \multicolumn{3}{c}{ Sexo Masculino } \\
& Genotipo & & Valor $p$ & \multicolumn{2}{c}{ Genotipo } & Valor $p$ \\
\hline & Del/Del & Ins/Del & Ins/Ins & & Del/Del & Ins/Del & Ins/Ins \\
CI (L) & $(\mathrm{n}: 3=8 \%)$ & $(\mathrm{n}: 23=62 \%)$ & $(\mathrm{n}: 11=30 \%)$ & & $(\mathrm{n}: 6=14 \%)$ & $(\mathrm{n}: 27=58 \%)$ & $(\mathrm{n}: 13=28 \%)$ \\
\hline
\end{tabular}

CI: capacidad inspiratoria; Ins: inserción; Del: deleción; (†): ANOVA; (¥); Kruskal-Wallis.

Tabla III. Capacidad Inspiratoria por genotipo dominante en jóvenes sedentarios de ambos sexos de la Universidad Católica del Maule, Chile.

\begin{tabular}{|c|c|c|c|c|c|c|}
\hline \multirow[b]{2}{*}{ Variable } & \multirow{2}{*}{\multicolumn{2}{|c|}{$\begin{array}{l}\text { Sexo Femenino } \\
\text { Genotipo }\end{array}$}} & \multicolumn{4}{|c|}{ Sexo Masculino } \\
\hline & & & Valor $p$ & & & Valor $p$ \\
\hline \multirow{3}{*}{ CI (L) } & $\begin{array}{c}\text { Ins/Del-Del/Del } \\
(\mathrm{n}: 26=70 \%)\end{array}$ & $\begin{array}{c}\text { Ins } / \operatorname{Ins} \\
(\mathrm{n}: 11=30 \%)\end{array}$ & & $\begin{array}{c}\text { Ins/Del-Del/Del } \\
(\mathrm{n}: 33=71 \%)\end{array}$ & $\begin{array}{c}\text { Ins } / \text { Ins } \\
(\mathrm{n}: 13=29 \%)\end{array}$ & \\
\hline & $2,76 \pm 0,06$ & $2,77 \pm 0,36$ & $0,877^{\mathfrak{f}}$ & $3,64 \pm 0,09$ & $4,00 \pm 0,14$ & $0,039^{f}$ \\
\hline & $\begin{array}{c}\text { Del/Ins-Ins/Ins } \\
\text { (n:34=92\%) }\end{array}$ & $\begin{array}{c}\text { Del/Del } \\
(\mathrm{n}: 3=8 \%)\end{array}$ & & $\begin{array}{c}\text { Del/Ins-Ins/Ins } \\
(\mathrm{n}: 40=87 \%)\end{array}$ & $\begin{array}{c}\text { Del/Del } \\
(\mathrm{n}: 6=13 \%)\end{array}$ & \\
\hline CI (L) & $2,80 \pm 0,05$ & $2,65 \pm 0,84$ & $0,029^{\mathfrak{f}}$ & $3,77 \pm 0,09$ & $3,59 \pm 0,10$ & $0,471^{ \pm}$ \\
\hline
\end{tabular}

CI: capacidad inspiratoria; Ins: inserción; Del: deleción; (£): t-student.

\section{DISCUSIÓN}

El objetivo de la presente investigación fue describir el comportamiento de la CI según genotipo del polimorfismo del gen de la ECA en jóvenes sedentarios. Previo a esto, se determinó la distribución genotípica del polimorfismo de este gen en la población de estudio. La muestra se encontró en equilibrio de Hardy Weinberg, lo que concuerda con resultados locales previos (Rosales et al.; Jalil et al., 1999). Del mismo modo, el análisis de los datos obtenidos evidenció una conducta normal de la CI, vale decir, fue significativamente mayor en hombres por sobre las mujeres. Este comportamiento concuerda con lo reportado por Lisboa et al. (2007), lo que se atribuye a las características antropométricas de ambos sexo, donde los hombres presentaron una talla, peso e IMC significativamente superior a las mujeres (Lisboa et al.).

Específicamente, al comparar el rendimiento de la CI entre genotipos del gen de la ECA, los datos obtenidos mostraron valores mayores en los hombres homocigotos Ins y en las mujeres con alelo dominante Ins, por tanto, este alelo se asociaría a un aumento de la CI. En experiencias previas, Contini et al. (2016) compararon el rendimiento ventilatorio en 100 sujetos con insuficiencia cardiaca. Sus resultados indican que no encontraron diferencias significativas en capacidad vital (CV) entre los diferentes genotipos (Contini et $a l$.). Esto discrepa de los resultados presentados donde existieron diferencias significativas asociadas al alelo Ins. Esta diferencia podría deberse a dos situaciones particulares: i) la muestra con la cual se realiza la comparación, la que presenta una patología asociada, mayor edad e IMC, situaciones que impactan en la función pulmonar (Peters et al., 2017); y ii) desconocimiento del real comportamiento de la $\mathrm{CI}$ en estos pacientes, debido a que la CV está compuesta por la CI más el volumen de reserva espiratorio. Por tanto, surge la duda de si la CI por sí sola podría haber tenido un comportamiento distinto (Lufti, 2017) a la muestra evaluada en esta investigación. 
Frente a la escasa información reportada, debemos señalar que Muñoz et al. (2017), demostraron que las mujeres universitarias sedentarias portadoras del alelo Ins tenían una mayor PIM en relación a las potadoras del alelo Del (Muñoz et al.). Si bien la PIM es un traductor de presión y no de volumen del sistema ventilatorio, se sabe que existe una directa relación entre ambos. Esto lo demostraron Pessoa et al. (2017) al relacionar ambas variables en adultos mayores, encontrando un $\mathrm{r}=0,97 ; \mathrm{p}<0,001$ entre PIM y CI, esto se debe a que en el recorrido axial del diafragma, su contracción permitiría reemplazar músculo por volumen de gas (Pessoa, 2017). La amplitud de movimiento a través de ultrasonografía, es una forma indirecta de medir movilidad y fuerza muscular diafragmática, permite determinar en tiempo real el desplazamiento de este músculo observándolo a través de una imagen, aquí un mayor cambio de amplitud representa mayor fuerza diafragmática (Connolly \& Mittendorfer, 2016) y CV (Boon \& O'Gorman, 2016). Esto es respaldado por Rocha et al., (2017), quienes investigaron el movimiento del diafragma asociado a la función pulmonar, encontrando un $\mathrm{r}=0,80$; $\mathrm{p}=0,001$ entre CI y movilidad del diafragma en milímetros (Rocha et al.). Aquí, el diafragma contribuye con aumento de volumen a través de un desplazamiento similar al de un pistón, donde el movimiento de la cúpula es axial y toda la zona de aposición en inspiración (acortamiento de las fibras musculares) es reemplazada por aire (De Troyer, 1997). Del mismo modo, en reconstrucciones tridimensionales se ha observado que el radio de curvatura del diafragma aumenta o disminuye significativamente con los cambios en los volúmenes pulmonares, tanto en el plano sagital como coronal, siendo este fenomeno más acentuado en el hemidiafragma izquierdo que el derecho. Dichos cambios en las configuraciones se sustentan en la ley de Laplace, en la cual la presión transdiafragmática es proporcional a la tensión muscular e inversamente proporcional al radio del cuadrado del músculo (Connolly \& Mittendorfer; Boon \& O'Gorman; De Troyer).

Además de esto, debemos considerar la acción de la ECA sobre el músculo esquelético. Al respecto, Dietze \& Henriksen (2008) describen que al inicio del ejercicio la contracción de fibras lentas y su rápida oxidación, son generadas por el sistema nervioso colinérgico. Este incremento bioenergético estimula la producción de kininas e inhibe la actividad de la ECA en el tejido endotelial del vaso sanguíneo, reduciendo la generación de angiotensina II. La kinina unida a los receptores b2 en el endotelio capilar y sarcolema de la fibra muscular, facilita la transmisión de óxido nítrico y prostaglandinas. Esto genera permeabilidad capilar, vasodilatación y el tránsito de glucosa a la miofibrilla (Dietze \& Henriksen). Este mecanismo favorece al diafragma, músculo con un $65 \%$ de fibras tipo I, hecho que se refleja en los datos obtenidos donde los los sujetos con alelo Ins tienen mayor PIM (Muñoz et al.) y CI.

Este estudio cuenta con limitaciones asociadas a la representatividad de muestra estudiada, por lo que la aplicabilidad de los hallazgos es en sujetos de las mismas características, asimismo, el grupo Del/Del del sexo femenino y masculino, contaron con un tamaño muestral pequeño, por lo que para futuras investigaciones sería importante considerar un mayor número de sujetos para este grupo, para poder potenciar las conclusiones encontradas. Además de esto, los argumentos presentados son mayormente en torno al desplazamiento del diafragma y el espacio que genera para el aumento de los volúmenes pulmonares. En este contexto, creemos necesario completar este estudio con mediciones imagenológicas como la ultrasonografía o la tomografía axial computarizada. Por otra parte, la escasa información reportada, abre el espacio a futuras investigaciones sobre la relación entre el polimorfismo de la ECA y los volúmenes pulmones.

En conclusión, existió una mayor CI en participantes de sexo femenino con dominancia alélica Ins y masculino homocigotos Ins, del gen de la ECA.

MUÑOZ, C. R.; MUÑOZ, M.; CONEI, V. D.; DEL SOL, M. \& PACHECO, V. A. Inspiratory capacity and polymorphism of angiotensin coverter enzime in young chileans. Int. J. Morphol., 36(3):1149-1153, 2018.

SUMMARY: The aim of the present research is to determine the inspiratory capacity (IC) in a group of sedentary Chilean university students grouped by the genotype (polymorphism rs4646994) of the angiotensin-converting enzyme (ACE) gene. Eighty three individuals (18 to 35 years old), 37 women and 46 men were selected through non-probabilistic sampling. The IC was measured through body plethysmograph, DNA was extracted from blood with EDTA and the polymerase chain reaction (PCR) was performed for the Ins / Del polymorphism of the ECA. Normality of the data was determined, using Student's t-test or ANOVA for the parametric variables, and in the non-parametric U-tests of Mann-Whitney or Kruskal-Wallis, a value of $\mathrm{p}<0.05$ being considered significant. The distribution of genotypes was found in Hardy Weinberg equilibrium $\left(X^{2}=1.872, p=0.171\right.$ for men and $X^{2}=3.424, p=0.064$ for women $)$, the IC, in both sexes, showed no significant differences when compared by genotype. The men carrying the Ins / Ins genotype had higher IC levels in relation to the gentile group Del / Del - Ins / Del. Women with allelic dominance Ins had a higher IC than those belonging to the Del / Del group. There was a greater IC in female participants with allelic dominance Ins and masculine homozygous Ins, of the ACE gene.

KEY WORDS: Inspiratory capacity; Angiotensin converting enzyme; Sedentary. 


\section{REFERENCIAS BIBLIOGRÁFICAS}

American Thoracic Society: Standardization of spirometry. Eur. Respir. J., 26(2):319-38, 2005.

Boon, A. J. \& O'Gorman, C. Ultrasound in the assessment of respiration. J. Clin. Neurophysiol., 33(2):112-9, 2016.

Caló, M. C. \& Vona, G. Gene polymorphisms and elite athletic performance. J. Anthropol. Sci., 86:113-31, 2008.

Connolly, A. M. \& Mittendorfer, B. Tracking diaphragm movement by using ultrasound to assess its strength. J. Physiol., 594(24):7147-8, 2016.

Contini, M.; Compagnino, E.; Cattadori, G.; Magrì, D.; Camera, M.; Apostolo, A.; Farina, S.; Palermo, P.; Gertow, K.; Tremoli, E.; Fiorentini, C. \& Agostoni, P. ACE-inhibition benefit on lung function in heart failure is modulated by ACE Insertion/Deletion polymorphism. Cardiovasc. Drugs Ther, 30(2):159-68, 2016.

De Troyer, A. Effect of hyperinflation on the diaphragm. Eur. Respir. J., 10(3):708-13, 1997.

Dietze, G. J. \& Henriksen, E. J. Angiotensin-converting enzyme in skeletal muscle: sentinel of blood pressure control and glucose homeostasis. $J$. Renin Angiotensin Aldosterone Syst., 9(2):75-88, 2008.

Jalil, J. E.; Piddo, A. M.; Cordova, S.; Chamorro, G.; Braun, S.; Jalil, R.; Vega, J.; Jadue'P, L.; Lavandero, S.; \& Lastra, P. Prevalence of the angiotensin I converting enzyme insertion/deletion polymorphism, plasma angiotensin converting enzyme activity, and left ventricular mass in a normotensive Chilean population. Am. J. Hypertens., 12(7):697704, 1999.

Lisboa, C.; Leiva, A.; Pinochet, R.; Repetto, P.; Borzone, G. \& Díaz, O. Valores de referencia de la capacidad inspiratoria en sujetos sanos no fumadores mayores de 50 años. Arch. Bronconeumol., 43(9):485-9, 2007.

Lufti, M. F. The physiological basis and clinical significance of lung volume measurements. Multidiscip. Respir. Med., 12(3):2-12, 2017.

Menezes, A .M.; Wehrmeister, F. C.; Hartwig, F. P.; Perez-Padilla, R.; Gigante, D. P.; Barros, F. C.; Oliveira, I. O.; Ferreira, G. D. \& Horta, B. L. African ancestry, lung function and the effect of genetics. Eur. Respir. J., 45(6):1582-9, 2015.

Muñoz, R.; Becerra, S. \& Pacheco, A. Influence of the polymorphism insertion / delection (rs4646994) of the angiotensin converting enzyme gene in the inspiratory and spiratory maximum pressure of chilean young sedentaries. Int. J. Morphol., 35(4):1254-60, 2017.

Nguyen Dinh Cat, A. \& Touyz, R. M. A new look at the renin-angiotensin system--focusing on the vascular system. Peptides, 32(10):2141-50, 2011

Peters, U.; Suratt, B. T.; Bates, J. H. T. \& Dixon, A. E. Beyond BMI: Obesity and lung diseases. Chest, (17)31:260-6, 2017.

Pessoa, M. F.; Brandão, D. C.; Sá, R. B.; Barcelar, J. M.; Rocha, T. D. S.; Souza, H. C. M. \& Dornelas de Andrade, A. Vibrating Platform Training Improves Respiratory Muscle Strength, Quality of Life, and Inspiratory Capacity in the Elderly Adults: A Randomized Controlled Trial. $J$. Gerontol. A. Biol. Sci. Med. Sci., 72(5):683-88, 2017.

Richter, M. J.; Gall, H.; Wittkämper, G.; Seeger, W.; Mayer, E.; Ghofrani, H. A.; Guth, S. \& Reichenberger, F. Inspiratory capacity is not altered in operable chronic thromboembolic pulmonary hypertension. Pulm. Circ., 7(2):543-46, 2017.

Rocha, T.; Souza, H.; Brandão, D. C.; Rattes, C.; Ribeiro, L.; Campos, S. L.; Aliverti, A. \& De Andrade, A. D. The Manual Diaphragm Release Technique improves diaphragmatic mobility, inspiratory capacity and exercise capacity in people with chronic obstructive pulmonary disease: a randomised trial. J. Physiother, 61(4):182-9, 2015.

Rosales, A.; Jaramillo, P.; Lanas, C.; Lanas, F. \& Salazar, L. Polimorfismo Ins/Del del gen de la enzima convertidora de angiotensina-I en individuos chilenos con enfermedad coronaria documentada por angiografía. Biomed. Sci., 1(1):20-7, 2009.

\author{
Dirección para correspondencia: \\ Rodrigo Muñoz Cofré \\ Universidad de La Frontera \\ Programa de Doctorado en Ciencias Morfológicas \\ Av. Francisco Salazar Nº 01145 \\ Temuco \\ CHILE
}

\section{E-mail: rodrigomunozcofre@gmail.com}

Recibido : 24-03-2018

Aceptado: 14-05-2018 